\title{
SPECTRAL PROPERTIES OF WICK POWER SERIES OF A FREE FIELD WITH AN INDEFINITE METRIC
}

\author{
A. G. Smirnov ${ }^{1}$, M. A. Soloviev"
}

\begin{abstract}
The properties of infinite series in the Wick powers of a free field whose two-point correlation function has a singular infrared behavior and does not satisfy the positivity condition are investigated. If these series are defined on an appropriate functional domain, then the fields to which they converge satisfy all conditions of the pseudo-Wightman formalism. For series convergent only on analytic test functions in the momentum representation, the spectral condition is formulated using the previously introduced notion of a carrier cone of an analytic functional. A suitable generalization of the Paley-Wiener-Schwartz theorem is used to prove that this condition is satisfied.
\end{abstract}

\section{Introduction}

In [1] a criterion was established that characterizes test functions such that averaging with these functions ensures the convergence of an infinite series in the Wick powers of a free field in a theory with an indefinite metric. We show here that if the functional domain for Wick series is so defined, then the fields to which they converge satisfy all conditions of the pseudo-Wightman formalism [2]. We also indicate a refinement of this formalism whose necessity follows from the analysis of the problem. The major difficulty lies in the correct statement of the spectral condition in the case when the representation of the translation group is pseudounitary and the series converges only on analytic test functions in the momentum representation, for which the usual formulation in terms of the spectral measure support or the distribution support becomes impossible. It is important that this case is rather typical than exceptional. For example, it includes the situation

\footnotetext{
${ }^{1}$ Lebedev Physics Institute, RAS, Moscow, Russia.
} 
with the normal exponential of the dipole ghost field [3, 4, which enters into the exact solutions of some gauge models. The problem of finding the corresponding generalization of the spectral condition was raised in [3]; it was suggested in [5] that it should be solved using the notion of a carrier cone (quasisupport) of an analytic functional, which had been successfully applied in nonlocal field theory. The results presented below show that this approach provides an exact description of the spectral properties of Wick series and can serve as a starting point for constructing a consistent Euclidean formulation of field theory with indefinite metric.

In Sec. 2, the properties of Wick series are analyzed in the traditional formalism, which applies if the functional domain of the series contains functions of compact support in both the coordinate and momentum representations. In Sec. 3, some mathematical tools required for analyzing the general case are introduced. To this end, we present certain facts concerning the Gelfand-Shilov spaces $S_{a}^{b}$ that generalize the more frequently used spaces $S_{\beta}^{\alpha}$, which were introduced by the same authors. The theory of the spaces $S_{a}^{b}$ was only outlined in [6]; here, we derive some new theorems for them which are needed for solving the problem under study and are also of independent interest. In Sec. 4, the Laplace transformation of analytic functionals on $S_{a}^{b}$ is considered. In Sec. 5, the precise formulation of the generalized spectral condition is given, and the proof is presented that it is satisfied for the sums of Wick series. The main tool in the proof is a Paley-WienerSchwartz-type theorem established in Sec. 4 for analytic functionals with an acute carrier cone. Section 6 is devoted to concluding remarks.

\section{Properties of Wick series in the traditional formal- ism}

Let $\phi$ be a neutral scalar free field acting in a pseudo-Hilbert state space $\mathcal{H}$, and let $d_{k}$ be the coefficients of a series in the Wick powers : $\phi^{k}$ : of this field. Let $D^{E}$ denote the linear span of the vacuum $\Psi_{0}$ and all vectors of the form

$$
\prod_{j=1}^{n}: \phi^{k_{j}}:\left(f_{j}\right) \Psi_{0}
$$

where the test functions $f_{j}$ run over a dense subspace $E$ in the Schwartz space $S$. It was proved in [1] that under some natural conditions on $d_{k}$ and for a suitably chosen $E$, the 
sequence of partial sums

$$
\varphi_{N}(f)=\sum_{k \leq N} d_{k}: \phi^{k}:(f) \quad(f \in E)
$$

with the domain $D^{E}$ has a strong graph limit $\varphi(f)$ which is an operator-valued distribution over $E$. Moreover, any Wick series subordinate to the one in question in the sense that its coefficients $d_{k}^{\prime}$ satisfy the inequality $\left|d_{k}^{\prime}\right| \leq C\left|d_{k}\right|$ is convergent, and all fields determined by such series have a common dense and invariant domain $D^{E}(\varphi) \subset \mathcal{H}$. Since the operator realization is constructed directly in the state space of the original free field $\phi$, a part of the conditions of general quantum field theory [2] hold for the field $\varphi$ in an obvious way. In particular, in view of the definition of strong graph limit [7], it follows from the mutual locality of Wick monomials that the field $\varphi$ is local and, moreover, mutually local with any field $\varphi^{\prime}$ determined by a subordinate series, i.e.,

$$
\left[\varphi(f), \varphi^{\prime}\left(f^{\prime}\right)\right] \Psi=0
$$

for any fixed test functions $f, f^{\prime} \in E$ with spacelike separated supports and for all $\Psi \in$ $D^{E}(\varphi)$. Originally, there is a pseudounitary representation of the Poincare group in the space $\mathcal{H}$. Its implementers $U(\xi, \Lambda)$ are defined on the cyclic domain $D_{0}$ of the field $\phi$ and transform it into itself. As is shown by simple examples, these operators are not necessarily bounded, but since they are pseudounitary, they are closable. By the construction of the Wick monomials, their closures are defined on the subspace $D^{S}$ and the more so on $D^{E}$. Let us consider a sequence $U(\xi, \Lambda) \varphi_{N}(f) U(\xi, \Lambda)^{-1} \Psi_{0}$, where $f \in E$. According to the transformation law for Wick monomials, which follows from that for $\phi$, this sequence coincides with $\varphi_{N}\left(f_{(\xi, \Lambda)}\right) \Psi_{0}$, where $f_{(\xi, \Lambda)}(x)=f\left(\Lambda^{-1}(x-\xi)\right)$. Passing to the limit as $N \rightarrow \infty$ and taking into account that the operator $U(\xi, \Lambda)$ is closable and the vacuum is invariant under it, we see that this operator can be extended to the vectors of the form $\varphi(f) \Psi_{0}$ in a one-to-one way and that it transforms them into $\varphi\left(f_{(\xi, \Lambda)}\right) \Psi_{0}$. Similarly, we have

$$
U(\xi, \Lambda) \varphi(f) \prod_{j=1}^{n} \varphi\left(f_{j}\right) \Psi_{0}=\varphi\left(f_{(\xi, \Lambda)}\right) \prod_{j=1}^{n} \varphi\left(f_{j(\xi, \Lambda)}\right) \Psi_{0}
$$

for test functions in $E$. As a consequence, the relation

$$
U(\xi, \Lambda) \varphi(f) U(\xi, \Lambda)^{-1} \Psi=\varphi\left(f_{(\xi, \Lambda)}\right) \Psi
$$


holds for any $\Psi \in D^{E}(\varphi)$, i.e., the Poincaré covariance condition is satisfied.

Let $\mathrm{d}$ be the space-time dimension. One of the natural conditions on the space $E$ is that the tensor product $E\left(\mathbb{R}^{\mathrm{d}}\right)^{\otimes n}$ should be dense in $E\left(\mathbb{R}^{n d}\right)$. In this case, the multilinear form $\Psi\left(f_{1}, \ldots, f_{n}\right)=\varphi\left(f_{1}\right) \ldots \varphi\left(f_{n}\right) \Psi_{0}$ uniquely determines a vector-valued distribution on $E\left(\mathbb{R}^{n \mathrm{~d}}\right)$. Indeed, let $f \in E\left(\mathbb{R}^{n \mathrm{~d}}\right)$, let $f_{\nu} \in E\left(\mathbb{R}^{\mathrm{d}}\right)^{\otimes n}$, and let $f_{\nu} \rightarrow f$ as $\nu \rightarrow \infty$. We have the relation

$$
\left\|\Psi\left(f_{\nu}\right)-\Psi\left(f_{\mu}\right)\right\|^{2}=T_{n}\left(\left(\overline{f_{\nu}-f_{\mu}}\right) \otimes\left(f_{\nu}-f_{\mu}\right)\right),
$$

where the distribution $T_{n}$ can be expressed in terms of the two-point Wightman function of the field $\phi$ and its Hilbert majorant (see [1]). The space $E$ is chosen in [1] in such a way that $T_{n}$ is defined and continuous on $E\left(\mathbb{R}^{2 n d}\right)$. Therefore $\left\|\Psi\left(f_{\nu}\right)-\Psi\left(f_{\mu}\right)\right\| \rightarrow 0$ as $\nu, \mu \rightarrow \infty$. Since the space $\mathcal{H}$ is complete, the Cauchy sequence $\Psi\left(f_{\nu}\right)$ converges in it to a vector, for which we use the standard notation

$$
\Psi(f)=\int \varphi\left(x_{1}\right) \ldots \varphi\left(x_{n}\right) f\left(x_{1}, \ldots, x_{n}\right) \mathrm{d} x_{1} \ldots \mathrm{d} x_{n} \Psi_{0} .
$$

The mapping $E\left(\mathbb{R}^{n d}\right) \rightarrow \mathcal{H}$ thus defined is linear and continuous. The representation $U(\xi, \Lambda)$ satisfies the spectral condition

$$
\operatorname{supp} \int\langle\Phi, U(\xi, I) \Psi\rangle e^{-i p \xi} \mathrm{d} \xi \subset \overline{\mathbb{V}}_{+},
$$

where $\mathbb{V}_{+}$is the upper light cone and the bar denotes closure. Here we originally deal with $\Phi, \Psi \in D_{0}$, but by the construction of Wick monomials, the condition (3) remains valid for the vectors in $D^{S}$ as well, and the matrix element under the integral sign remains polynomially bounded with respect to $\xi$ in this case. However, we stress that this boundedness property does not necessarily hold for arbitrary $\Phi$ and $\Psi$ belonging to the domain of the closure of the operator $U(\xi, I)$. If the Fourier transform $\mathcal{F}(E)$ of $E$ contains functions of compact support, then the spectral properties of the functional $\Psi(f)$ defined by (2) can be derived from (3) in the usual way. Namely, let $\Phi \in D^{E}(\varphi)$. The expression $\langle\Phi, U(\xi, I) \Psi(f)\rangle$ is the convolution of the test function $f$ and a distribution in $E^{\prime}$ (considered in the plane $x_{1}=\ldots=x_{n}=\xi$ ), and therefore its growth with respect to $\xi$ is limited by the indicator function defining the space $E$. Let $f_{1} \in E\left(\mathbb{R}^{d}\right)$. Then

$$
\int\langle\Phi, U(\xi, I) \Psi(f)\rangle f_{1}(\xi) \mathrm{d} \xi=\left\langle\Phi, \Psi\left(f_{2}\right)\right\rangle,
$$


where

$$
f_{2}\left(x_{1}, \ldots, x_{n}\right)=\int f\left(x_{1}-\xi, \ldots, x_{n}-\xi\right) f_{1}(\xi) \mathrm{d} \xi .
$$

The Fourier transform takes $f_{2}$ to

$$
\hat{f}_{2}=\int e^{i p x} f_{2}(x) \mathrm{d} x=\hat{f} \hat{f}_{1}\left(p_{1}+\ldots+p_{n}\right) .
$$

According to (阿) the integral (四) vanishes if supp $\hat{f}_{1} \cap \overline{\mathbb{V}}_{+}=\emptyset$. On the other hand, we have $\Psi(f) \stackrel{\text { def }}{=} \hat{\Psi}(\check{f})$, where

$$
\check{f}(p)=(2 \pi)^{-n \mathrm{~d}} \int e^{-i p x} f(x) \mathrm{d} x .
$$

Consequently, the support of $\hat{\Psi}$ with respect to the variable $p_{1}+\ldots+p_{n}$ is contained in $\overline{\mathbb{V}}_{-}$. The same is true for the variable $p_{m}+\ldots+p_{n}, m \leq n$, i.e., the functional (2) in the momentum space is supported by the cone

$$
K_{n-}=\left\{p \in \mathbb{R}^{n \mathrm{~d}}: p_{m}+\ldots+p_{n} \in \overline{\mathbb{V}}_{-}, \quad \forall m=1, \ldots, n\right\}
$$

In particular, this cone contains the support of the Fourier transform of the $n$-point Wightman function of the field $\varphi$, which is clear from its explicit expression via the twopoint function of the original field $\phi$. If $\mathcal{F}(E)$ consists of analytic functions, then the above derivation is no longer valid, and some other means of functional analysis are needed for describing the spectral properties.

\section{Gelfand-Shilov-Gurevich spaces}

It was shown in [1] that a suitable space $S_{a}^{b}$ can be taken as the functional domain $E$ of the Wick series $\sum d_{k}: \phi^{k}:(x)$. Such spaces are particularly convenient from the standpoint of the Fourier transformation, which simply interchanges the indices $a$ and $b$. The defining indices are sequences of positive numbers satisfying the regularity conditions

$$
a_{k+l} \leq C_{1} h_{1}^{k+l} a_{k} a_{l}, \quad b_{k+l} \leq C_{2} h_{2}^{k+l} b_{k} b_{l}
$$

where $C_{1,2}$ and $h_{1,2}$ are constants 2. The space $S_{b}^{a}=\mathcal{F}\left(S_{a}^{b}\right)$, which in the context of the problem under study is the test function space in the momentum representation, consists

\footnotetext{
${ }^{2}$ The conditions (6) ensure that the operations required for a convenient calculus are performable in $S_{a}^{b}$. It is noteworthy that the weaker conditions $a_{k+1} \leq C_{1} h_{1}^{k} a_{k}, \quad b_{k+1} \leq C_{2} h_{2}^{k} b_{k}$ proved to be sufficient for deriving the results in [1].
} 
of smooth functions satisfying the bounds

$$
\left|p^{\lambda} \partial^{\kappa} g(p)\right| \leq C A^{|\kappa|} B^{|\lambda|} a_{|\kappa|} b_{|\lambda|},
$$

where $\kappa$ and $\lambda$ are multi-indices whose norm is defined as the sum of the components and the constants $A, B$ and $C$ depend on $g$. The conditions (7) can be rewritten as

$$
b(|p| / B)\left|\partial^{\kappa} g(p)\right| \leq C A^{|\kappa|} a_{|\kappa|}
$$

where $b(s)=\sup _{l \in \mathbb{N}}\left(s^{l} / b_{l}\right),|p|=\max _{j}\left|p_{j}\right|$, and $\mathbb{N}$ denotes the set of nonnegative integers. The function $b(s)$ is called the indicator function. If $a_{k}^{1 / k}=O(k)$, then the elements of $S_{b}^{a}$ are analytic, and, as a rule, the indefinite metric Wick series converge only on such test functions. This means that the matrix elements of the fields determined by these series are analytic functionals in the momentum representation. A special role is played by the spaces defined by the sequence $a_{k}=k$ !, which are usually denoted by $S_{b}^{1}$. The elements of the dual space $S_{b}^{\prime 1}$ can be interpreted as hyperfunctions increasing at infinity no faster than $C_{\epsilon} b(\epsilon|p|)$, where $\epsilon$ is arbitrarily small. As is known, hyperfunctions form the broadest class of distributions for which the notion of support is well defined. It was shown in [8, 9] that a part of their properties are inherited by analytic functionals of the class $S_{b}^{\prime a}$, where $a_{k}=k^{\alpha k}, b_{l}=l^{\beta l}$ and the numbers $\alpha$ and $\beta$ satisfy the conditions $0 \leq \alpha<1$ and $\beta>1$. Here, we extend the theory developed in [8, 9] to a broader class of functionals.

Definition 1. Let $\alpha(s)$ and $\beta(s)$ be nonnegative continuous functions indefinitely increasing on the half-axis $s \geq 0$, let $\alpha(s)$ be convex and differentiable for $s>0$, and let $\beta(s)$ be convex with respect to $\ln s$ and satisfy the condition

$$
2 \beta(s) \leq \beta(h s)
$$

with a constant $h>1$. We define $\mathcal{E}_{\beta}^{\alpha}$ as the space consisting of entire analytic functions on $\mathbb{C}^{n}$ such that

$$
|g(p+i q)| \leq C \exp \{\alpha(A|q|)-\beta(|p| / B)\},
$$

where the constants $A, B$ and $C$ depend on $g$.

We write $\alpha_{1} \prec \alpha$ if there are constants $C$ and $H$ such that $\alpha_{1}(s) \leq C+\alpha(H s)$.

Theorem 1. Let $\beta \prec \alpha$. Then $\mathcal{E}_{\beta}^{\alpha}$ coincides with the space $S_{b}^{a}\left(\mathbb{R}^{n}\right)$ determined by the sequences

$$
a_{k}=\sup _{r \geq 0} r^{k} e^{-\alpha_{*}(r)} \quad b_{l}=\sup _{s \geq 0} s^{l} e^{-\beta(s)}
$$


where $\alpha_{*}(r)=\sup _{s>0}(r s-\alpha(s))$.

The function $\alpha_{*}$ is said to be monotonically conjugate to the convex function $\alpha$ (see [10). The conditions (6) hold for the sequences (11) in view of (9) and the inequality $2 \alpha_{*}(s) \leq \alpha_{*}(2 s)$, which follows from the convexity of $\alpha_{*}$ under the normalization condition $\alpha(0)=0$.

To prove Theorem 1, we need the following three lemmas.

Lemma 1. The relation

$$
\sup _{r \geq 0} r^{k} e^{-\alpha_{*}(r)}=(k / e)^{k} \inf _{s>0} s^{-k} e^{\alpha(s)} .
$$

holds for any function $\alpha(s)$ satisfying the conditions of Definition 1 and for all $k \geq 0$.

Proof. The expression in the right-hand side is immediately recovered, if we substitute $e^{-\alpha_{*}(r)}=\inf _{s>0} e^{\alpha(s)-r s}$ in the left-hand side of (12) and change the order in which the supremum and the infimum are calculated. Since $\sup _{r} \inf _{s} G(r, s) \leq \inf _{s} \sup _{r} G(r, s)$ for any function $G$, it is only necessary to show that the opposite inequality also holds in the case under consideration, i.e., to prove (according to the standard terminology [10]) that the function $G(r, s)=r^{k} e^{\alpha(s)-r s}$ has a saddle value. The validity of (12) with $k=0$ is obvious; we therefore suppose that $k>0$. We recall that a differentiable convex function is continuously differentiable. The point $s_{k}$ at which $\inf _{s>0} s^{-k} e^{\alpha(s)}$ is attained is determined by the equation $\alpha^{\prime}(s)=k / s$, whose solution is unique because the function $\alpha^{\prime}$ is nonnegative, monotonic, and not identically zero. Set $r_{k}=\alpha^{\prime}\left(s_{k}\right)$. We have

$$
\sup _{r \geq 0} \inf _{s>0} r^{k} e^{\alpha(s)-r s} \geq \inf _{s>0} r_{k}^{k} e^{\alpha(s)-r_{k} s}=\left(k / e s_{k}\right)^{k} e^{\alpha\left(s_{k}\right)}
$$

because $\alpha(s)-r_{k} s$ is a convex function and any of its stationary points is the point of absolute minimum. The lemma is thus proved.

Lemma 2. For any function $\beta(s)$ satisfying the conditions of Definition 1, and for any $\epsilon>0$ there is a constant $C_{\epsilon}$ such that

$$
\beta(s)+\ln s \leq C_{\epsilon}+\beta((1+\epsilon) s) .
$$

Proof. For any nondecreasing function $\beta$ which is convex with respect to $\ln s$ and is not identically constant, there is a number $c$ such that

$$
\beta(s) \geq c \ln s
$$


for sufficiently large $s$. If (9) holds in addition, then (15) is true for arbitrarily large $c$. The convexity with respect to $\ln s$ implies

$$
\beta(h s) \leq \tau \beta\left(h^{1 / \tau} s\right)+(1-\tau) \beta(s)
$$

for $0 \leq \tau \leq 1$. Set $\tau=N /(N+1)$ and take $N$ such that $h^{1 / N}<1+\epsilon$. Then

$$
\beta(h s)-\beta(s) \leq N[\beta((1+\epsilon) h s)-\beta(h s)]
$$

Here we have $\beta(h s)-\beta(s) \geq N \ln s-C_{N}$ in view of the condition (9) and the inequality (15) with $c=N$. After the change of notation $h s \rightarrow s$, we obtain (14).

Lemma 3. Let the function $\beta(s)$ satisfy the conditions of Definition 1 , and let the sequence $b_{l}$ be determined by the relations (11) and $b(s)=\sup _{l \in \mathbb{N}}\left(s^{l} / b_{l}\right)$. Then

$$
b(s) \leq e^{\beta(s)} \leq C_{\epsilon}^{\prime} b((1+\epsilon) s)
$$

for any $\epsilon>0$.

Proof. Only the right-hand inequality is nontrivial here. We temporarily suppose that $l$ is an arbitrary real number, not necessarily a positive integer, and note that the function $b_{l}$ is nondecreasing for $l>\beta(e)$ because the infimum in definition (11) is attained at $s>1$ in this case. The function $\ln b_{l}$ is conjugate to $\beta\left(e^{t}\right)$ and is finite for $l \geq 0$. The operation of conjugation is involutory on convex closed functions (see [10]), and therefore $\sup _{l \geq 0}\left(s^{l} / b_{l}\right)=e^{\beta(s)}$. If $s$ is sufficiently large, then the supremum is attained here at large $l$ because $\beta(s)$ increases faster than $c \ln s$ with arbitrary $c$. Let $[l]$ be the integer part of $l$. Then

$$
b(s) \geq s^{[l]} / b_{[l]} \geq s^{l-1} / b_{l}=e^{\beta(s)-\ln (s)},
$$

and the application of (14) completes the proof.

Proof of Theorem 1. Let $g \in \mathcal{E}_{\beta}^{\alpha}$. For the natural choice of the topology on $\mathcal{E}_{\beta}^{\alpha}$, the least constant $C$ for which (10) is true is the norm $\|g\|_{A, B}$. Let $\mathcal{D}(s)$ and $\partial_{0} \mathcal{D}(s)$ denote the polydisk $\left\{\zeta \in \mathbb{C}^{n}:|\zeta|=\max _{j}\left|\zeta_{j}\right| \leq s\right\}$ and its skeleton. Applying the Cauchy formula, using the arbitrariness in the choice of $s$, and taking into account the inequality $\beta(|p| / 2) \leq \beta(|\zeta+p|)+\beta(|\zeta|)$ implied by the monotonicity and nonnegativity of $\beta$, we obtain

$$
\left|p^{\lambda} \partial^{\kappa} g(p)\right|=\left|p^{\lambda}\right| \frac{\kappa !}{(2 \pi)^{n}}\left|\int_{\partial_{0} \mathcal{D}(s)} \frac{g(\zeta+p)}{\zeta^{\kappa+I}} \mathrm{~d} \zeta\right| \leq\|g\|_{A, B}|p|^{|\lambda|} e^{-\beta(|p| / 2 B)}|\kappa| ! \inf _{s>0} s^{-|\kappa|} e^{\alpha(A s)+\beta(s / B)}
$$




$$
\leq C_{\epsilon}\|g\|_{A, B}(2(A+H / B+\epsilon))^{|\kappa|}(2 B)^{|\lambda|} a_{|\kappa|} b_{|\lambda|},
$$

where the right-hand inequality is derived using the Stirling formula, Lemma 1, the condition $\beta \prec \alpha$, and the inequality $\alpha\left(s_{1}\right)+\alpha\left(s_{2}\right) \leq \alpha\left(2\left(s_{1}+s_{2}\right)\right.$. Hence, the space $\mathcal{E}_{\beta}^{\alpha}$ is continuously embedded in $S_{b}^{a}$.

Conversely, let $g \in S_{b}^{a}$. The norms in $S_{a}^{b}$ will be marked with the prime. Using the Taylor formula, the condition (8), and the inequality $|\kappa| ! \leq n^{|\kappa|} \kappa !$, we obtain the estimate

$$
|g(p+i q)| \leq \sum_{\kappa \in \mathbb{N}^{n}} \frac{\left|q^{\kappa}\right|}{\kappa !}\left|\partial^{\kappa} g(p)\right| \leq C_{\epsilon}\|g\|_{A, B}^{\prime} \frac{1}{b(|p| / B)} \sup _{k \in \mathbb{N}}(n(A+\epsilon)|q|)^{k} \frac{a_{k}}{k !}
$$

for the analytic continuation of $g$ into $\mathbb{C}^{n}$. By Lemma 1 and the inequality $1 / k ! \leq e^{k} / k^{k}$, we have $a_{k} / k ! \leq e^{\alpha(s)} / s^{k}$ for any $s$. Therefore, the supremum with respect to $k$ can be majorized by the function $e^{\alpha(n(A+\epsilon)|q|)}$. Applying Lemma 3 completes the proof.

Remark. It is well known that the space $S_{b}^{1}$ can be similarly redefined. It can be regarded as being defined by the discontinuous convex function $\alpha(s)$ vanishing for $0 \leq s \leq 1$ and equal to $+\infty$ for $s>1$, and by the function $\beta(s)=\ln b(s)$. Accordingly, its elements are analytic in the domains of the form $|y|<1 / A$.

The subclass of the spaces of type $S$ characterized by Theorem 1 will be called the Gelfand-Shilov-Gurevich spaces because, in some respects, they are close to the spaces of type $W$ introduced by Gurevich. The further construction of the theory follows that in [8, 9], and instead of presenting complete proofs, we comment on their main points. The key step consists in introducing, in addition to $\mathcal{E}_{\beta}^{\alpha}$, the kindred spaces over cones.

Definition 2. Let $U$ be an open cone in $\mathbb{R}^{n}$, and let the functions $\alpha$ and $\beta$ satisfy the conditions of Definition 1. The space $\mathcal{E}_{\beta}^{\alpha}(U)$ consists of entire functions on $\mathbb{C}^{n}$ possessing the boundedness property

$$
|g(p+i q)| \leq C \exp \left\{\alpha(A|q|)+\alpha \circ \delta_{U}(A p)-\beta(|p| / B)\right\},
$$

where $\delta_{U}(p)$ is the distance from the point $p$ to the cone $U$ and the constants $A, B$ and $C$ depend $g$.

Theorem 2. If the space $\mathcal{E}_{\beta}^{\alpha}(U)$ is endowed by the inductive-limit topology with respect to the family of the Banach spaces $\mathcal{E}_{\beta, B}^{\alpha, A}(U)$ of entire functions with the norms

$$
\|g\|_{U, A, B}=\sup _{p, q}|g(p+i q)| \exp \left\{-\alpha(A|q|)-\alpha \circ \delta_{U}(A p)+\beta(|p| / B)\right\}
$$


then $\mathcal{E}_{\beta}^{\alpha}(U)$ becomes a DFS spac£ and is consequently a complete, Hausdorff, barrelled, reflexive, separable, and Montel space.

We recall that DFS spaces are limits of injective compact sequences of locally convex spaces. For an arbitrary sequence contained in the unit ball of $\mathcal{E}_{\beta, B}^{\alpha, A}(U)$, the application of the Montel theorem and the diagonalization process permit separating a subsequence uniformly converging on compact sets in $\mathbb{C}^{n}$ to an element of this space. In the broader space $\mathcal{E}_{\beta, B^{\prime}}^{\alpha, A^{\prime}}(U), A^{\prime}>A, B^{\prime}>B$, this convergence holds with respect to the norm because

$$
\lim _{|q| \rightarrow \infty} e^{\alpha(A|q|)-\alpha\left(A^{\prime}|q|\right)}=0, \quad \lim _{|p| \rightarrow \infty} e^{-\beta(|p| / B)+\beta\left(|p| / B^{\prime}\right)}=0
$$

by virtue of Lemma 2 . Therefore, the canonical mapping $\mathcal{E}_{\beta, B}^{\alpha, A}(U) \rightarrow \mathcal{E}_{\beta, B^{\prime}}^{\alpha, A^{\prime}}(U)$ is compact, which precisely proves Theorem 2 . For the same reason, $\mathcal{E}_{\beta, B+}^{\alpha, A+}(U)=\bigcap_{A^{\prime}>A, B^{\prime}>B} \mathcal{E}_{\beta, B^{\prime}}^{\alpha, A^{\prime}}(U)$ is a Frechét space, which is essential for the derivation of the result below.

Theorem 3. For any pair $U_{1}, U_{2} \subset \mathbb{R}^{n}$ of open cones, the relation

$$
\mathcal{E}_{\beta}^{\alpha}\left(U_{1}\right) \hat{\otimes}_{i} \mathcal{E}_{\beta}^{\alpha}\left(U_{2}\right)=\mathcal{E}_{\beta}^{\alpha}\left(U_{1} \times U_{2}\right)
$$

holds, where the subscript $i$ means that the tensor product is endowed with the inductive topology and the hat denotes completion.

The proof of this theorem is the same as that of Theorem 3 in [9]. According to the Carleman-Ostrowski theorem on quasianalyticity conditions, the space $\mathcal{F}\left(\mathcal{E}_{\beta}^{\alpha}\right)$ contains functions of compact support if and only if

$$
\int_{1}^{\infty} \frac{\beta(s)}{s^{2}} \mathrm{~d} s<\infty
$$

We assume that the condition (20) holds because the formalism we develop is intended for application to the local field theory.

Theorem 4. Under the condition (20), the space $\mathcal{E}_{\beta}^{\alpha}$ is dense in each of the spaces $\mathcal{E}_{\beta}^{\alpha}(U)$, where $U$ is an open cone.

This theorem is proved in the same way as its particular case in [9]. A much simpler proof is possible under the additional assumption that the space $\mathcal{E}_{\alpha}^{\alpha}$ is nontrivial, which suffices for the majority of applications we are interested in. Namely, taking a function

\footnotetext{
${ }^{3}$ Here DFS is the standard notation for the class of topological vector spaces dual to the FrechétSchwartz spaces.
} 
$e_{0} \in \mathcal{E}_{\alpha}^{\alpha}$ possessing the property $\int e_{0}(p) \mathrm{d} p=1$, we can approximate any element $g \in \mathcal{E}_{\beta}^{\alpha}(U)$ with functions $g_{\nu} \in \mathcal{E}_{\alpha}^{\alpha}$ by setting $g_{\nu}=e_{\nu} g$, where $e_{\nu}$ is a sequence of Riemann sums for the integral $\int e_{0}(p-\eta) \mathrm{d} \eta$ or, more explicitly,

$$
e_{\nu}(p)=\sum_{\kappa \in \mathbb{Z}^{n},|\kappa|<\nu^{2}} e_{0}(p-\kappa / \nu) \nu^{-n}
$$

A closed cone $K \subset \mathbb{R}^{n}$ is called a carrier cone of a functional $u \in \mathcal{E}_{\beta}^{\prime \alpha}$, if $u$ can be continuously extended to every space $\mathcal{E}_{\beta}^{\alpha}(U)$, where $U \supset K \backslash\{0\}$ or, in other words, if $u$ can be continuously extended to the union

$$
\mathcal{E}_{\beta}^{\alpha}(K)=\bigcup_{U \supset K \backslash\{0\}} \mathcal{E}_{\beta}^{\alpha}(U)
$$

endowed with the inductive-limit topology.

Theorem 5. Any functional $u \in \mathcal{E}_{\beta}^{\prime \alpha}$ with a carrier cone $K_{1} \cup K_{2}$ can be decomposed as $u=u_{1}+u_{2}$, where $u_{j} \in \mathcal{E}_{\beta}^{\prime \alpha}\left(K_{j}\right), \quad j=1,2$.

Theorem 6. If each of the cones $K_{1}$ and $K_{2}$ is a carrier cone of a functional $u \in \mathcal{E}_{\beta}^{\prime \alpha}$, then $K_{1} \cap K_{2}$ is also its carrier cone.

As a consequence, there exists the smallest closed cone $K$ such that $u \in \mathcal{E}_{\beta}^{\prime \alpha}(K)$. It can be called the quasisupport of the functional $u$.

The spaces $\mathcal{E}_{\beta}^{\alpha}(K)$ inherit the topological properties specified in Theorem 2. Therefore, Theorems 5 and 6 are equivalent to the corresponding dual assertions for test functions, and among these assertions, only the one concerning the possibility of decomposing any function $g \in \mathcal{E}_{\beta}^{\alpha}\left(K_{1} \cap K_{2}\right)$ into a sum of functions belonging to $\mathcal{E}_{\beta}^{\alpha}\left(K_{1}\right)$ and $\mathcal{E}_{\beta}^{\alpha}\left(K_{2}\right)$ needs a proof. If $\mathcal{E}_{\alpha}^{\alpha}$ is nontrivial, then such a decomposition presents no problems. Namely, let $g \in \mathcal{E}_{\beta, B}^{\alpha, A}(U)$, where $U$ is a conic neighborhood of the intersection $K_{1} \cap K_{2}$, and let $W$ be its slightly smaller neighborhood such that $\bar{W} \backslash\{0\} \subset U$. Since the angular distance between the closed cones $K_{j} \backslash W$ is nonzero, there exist their conic neighborhoods $W_{j}$ and a number $\theta>0$ such that

$$
|p-\eta| \geq \theta|p|, \quad|p-\eta| \geq \theta|\eta|
$$

for all $p \in W_{1}$ and $\eta \in W_{2}$. We take an arbitrary function $e_{0} \in \mathcal{E}_{\alpha, B_{0}}^{\alpha, A_{0}}$ with the property $\int e_{0}(p) \mathrm{d} p=1$ and set

$$
e(p)=\int_{W_{2}} e_{0}(p-\eta) \mathrm{d} \eta
$$


Using the convexity of $\alpha$ and the inequalities (21), we can verify that $e g \in \mathcal{E}_{\beta}^{\alpha}\left(K_{1}\right)$ given

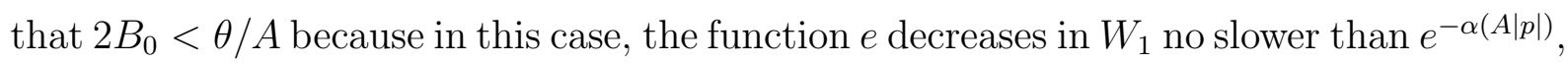
which suppresses the growth of $g$ in this cone. Similarly, we have $(1-e) g \in \mathcal{E}_{\beta}^{\alpha}\left(K_{2}\right)$ for $2 B_{0}<\theta^{\prime} / A$, where $\theta^{\prime}$ is the angular distance from $K_{2} \backslash W$ to the complement of $W_{2}$. If $\mathcal{E}_{\alpha}^{\alpha}$ is trivial, then the desired decomposition can be derived using the standard method for solving Cousin problems using the Hörmander $L^{2}$-estimates (see [8]).

\section{Fourier-Laplace transformation in $\mathcal{E}_{\beta}^{\prime \alpha}$}

We proceed to extend the theory of the Fourier-Laplace transformation to functionals of the class $\mathcal{E}_{\beta}^{\prime \alpha}$. The nonquasianalyticity condition (20) implies the inequality $\beta(s)<C_{\epsilon}+\epsilon s$, where $\epsilon$ is arbitrarily small. Therefore, the function

$$
\beta^{*}(t)=\inf _{s>0}(s t-\beta(s))
$$

is defined on the half-axis $t>0$. We note that it is nonpositive, concave, and monotonic and that it tends to $-\infty$ as $t \rightarrow 0$.

7. Let $K$ be an acute cone and $V$ be the interior of its dual cone $K^{*}=\{y: p y \geq$ $0, \forall p \in K\}$. If the condition (20) holds, then the Laplace transform $\mathbf{v}(z)=\left(u, e^{i(\cdot, z)}\right)$ of any functional $u \in \mathcal{E}_{\beta}^{\prime \alpha}(K)$ is holomorphic in the domain $T^{V}=\{z=x+i y: y \in V\}$ and satisfies the inequality

$$
|\mathbf{v}(z)| \leq C_{\epsilon}\left(V^{\prime}\right) \exp \left\{\alpha_{*}(\epsilon|z|)-\beta^{*}(|y| / \epsilon)\right\} \quad\left(y \in V^{\prime}\right)
$$

for an arbitrary $\epsilon>0$ and for every compact subcone $V^{\prime}$ of $V$ (i.e., such that $\bar{V}^{\prime} \backslash\{0\} \subset V$ ). As $y \rightarrow 0$ inside a fixed cone $V^{\prime}$, the function $\mathbf{v}(x+i y)$ tends to the Fourier transform of the functional $u$ in the topology of the space $\mathcal{F}\left(\mathcal{E}_{\beta}^{\prime \alpha}\right)$.

Proof. We have

$$
|\mathbf{v}(z)| \leq\|u\|_{U, A, B}\left\|e^{i(p+i q) z}\right\|_{U, A, B}
$$

for any $A, B>0$ and every $U \supset K \backslash\{0\}$, where

$$
\left\|e^{i(p+i q) z}\right\|_{U, A, B}=\sup _{p} \exp \left\{-p y-\alpha \circ \delta_{U}(A p)+\beta(|p| / B)\right\} \sup _{q} \exp \{-q x-\alpha(A|q|)\} .
$$

In what follows, it is convenient to regard $|\cdot|$ as the Euclidean norm in $\mathbb{R}^{n}$. The supremum with respect to $q$ in (24) with $A>1 / \epsilon$ is majorized by the function $e^{\alpha_{*}(\epsilon|x|)}$. We take a 
cone $U$ that is compact in another cone $U^{\prime}$ that is compact in $\operatorname{int} V^{* *}$. If $p \notin U^{\prime}$, then $\delta_{U}(p)>\theta|p|$, where $\theta>0$, and consequently $\alpha \circ \delta_{U}(A p)>\alpha(A \theta|p|)$. Moreover, we have $\beta(|p| / B)<C+|p| / B$, and the supremum with respect to $p \notin U^{\prime}$ for $2 A \theta>1 / \epsilon$ therefore does not exceed $C_{\epsilon} e^{\alpha_{*}(\epsilon|y|)}$. If $p \in U^{\prime}$, then the inclusion $\bar{U}^{\prime} \backslash\{0\} \subset$ int $V^{\prime *}$ implies that $-p y \leq-\theta^{\prime}|p||y|$ for all $y \in V^{\prime}$. Hence, the supremum with respect to $p \in U^{\prime}$ for $B \theta^{\prime}>1 / \epsilon$ does not exceed $-\beta^{*}(|y| / \epsilon)$. The estimate (23) is thus proved. It is similarly established that the difference quotients corresponding to the partial derivatives $\partial e^{i(p+i q) z} / \partial z_{j}$ converge to them for any $z \in T^{V}$ in the topology of $\mathcal{E}_{\beta}^{\alpha}(U)$, and $\mathbf{v}(z)$ is consequently holomorphic for $z \in T^{V}$. By Theorem 1, a function $f \in \mathcal{F}\left(\mathcal{E}_{\beta}^{\alpha}\right)=S_{a}^{b}$ satisfies the inequality $|f(x)| \leq C / a(|x| / A)$, where $a(r)=\sup _{k}\left(r^{k} / a_{k}\right) \geq c_{\varepsilon} e^{\alpha_{*}(r /(1+\varepsilon))}$ according to Lemma 3 applied to $\alpha_{*}$. Therefore, for every $y \in V^{\prime}$, the function $f$ is integrable with the function $\left(u, e^{i(\cdot, z)}\right)$ not only for the element $u$ under consideration but also for any $u \in \mathcal{E}_{\beta}^{\prime \alpha}(U)$. Consequently, the sequence of Riemann sums for the integral

$$
\int e^{i z(p+i q)} f(x) \mathrm{d} x
$$

is weakly Cauchy in the space $\mathcal{E}_{\beta}^{\alpha}(U)$, and because this is a Montel space, it is convergent in $\mathcal{E}_{\beta}^{\alpha}(U)$. Hence,

$$
\int \mathbf{v}(x+i y) f(x) \mathrm{d} x=\left(u, e^{-(\cdot, y)} g\right)
$$

where

$$
g(\zeta)=\int e^{i \zeta x} f(x) \mathrm{d} x \quad(\zeta=p+i q) .
$$

It remains to prove that $e^{-\zeta y} g(\zeta) \rightarrow g(\zeta)$ in the topology of $\mathcal{E}_{\beta}^{\alpha}(U)$ as $y \rightarrow 0$ inside $V^{\prime}$. By the first mean value theorem, we have $\left|e^{-\zeta y}-1\right| \leq|\zeta||y| \max _{0 \leq t \leq 1} e^{-p y t}$. If $g \in \mathcal{E}_{\beta, B_{0}}^{\alpha, A_{0}}$, then we obtain

$$
\left\|\left(e^{-\zeta y}-1\right) g\right\|_{U, A, B} \leq C|y| \max _{0 \leq t \leq 1} \sup _{p} \exp \left\{-p y t-\alpha \circ \delta_{U}(A p)\right\}
$$

for $A>A_{0}$ and $B>B_{0}$. Here, the expression $-p y t-\alpha \circ \delta_{U}(A p)$ in the exponential function is negative for $p \in U^{\prime}$ and $y \in V^{\prime}$ and does not exceed $\alpha_{*}(|y| / A \theta)$ for $p \notin U^{\prime}$. The theorem is proved.

Let $V$ be an open connected cone in $\mathbb{R}^{n}$, and let $\mathcal{A}_{\alpha_{*}}^{\beta^{*}}(V)$ denote the space of holomorphic functions in $T^{V}$ and satisfying the condition (23). For the natural choice of topology, it is a topological algebra with respect to multiplication because the condition (9) implies 
that $2 \beta^{*}(t) \geq \beta^{*}(2 t / h)$. The most important for us and at the same time the most difficult problem is to generalize the Paley-Wiener-Schwartz theorem to analytic functionals of the class $\mathcal{E}_{\beta}^{\prime \alpha}$. We do this under an additional condition on the growth of the elements of $\mathcal{A}_{\alpha_{*}}^{\beta^{*}}(V)$ at infinity. This condition is very weak, and it holds for the applications we are interested in. We say that two functions $\beta$ and $\beta_{1}$ are equivalent if $\beta_{1} \prec \beta$ and $\beta \prec \beta_{1}$. In this case, they specify the same space.

Theorem 8. Let the functions $\alpha$ and $\beta$ possess the properties specified in Definition 1 , let the conjugate function $\alpha_{*}$ of $\alpha$ increase no faster than a first-order finite-type exponential function, and let $\beta$ satisfy the condition (20) and be equivalent to a continuous concave function. Then the Laplace transformation establishes an isomorphism between the space $\mathcal{E}_{\beta}^{\prime \alpha}\left(V^{*}\right)$ and the algebra $\mathcal{A}_{\alpha_{*}^{*}}^{\beta^{*}}(V)$ for any open connected cone $V$.

Proof. The cone $V$ lies inside $V^{* *}$. The Laplace transform of the space $\mathcal{E}_{\beta}^{\prime \alpha}\left(V^{*}\right)$ is therefore contained in $\mathcal{A}_{\alpha_{*}}^{\beta^{*}}(V)$ by Theorem 7 , and the problem consists in proving the inverse inclusion. As before, let the sequence $a_{k}$ be defined by the first formula in (11). Any function $\mathbf{v} \in \mathcal{A}_{\alpha_{*}}^{\beta^{*}}(V)$ has a boundary value in the sense of hyperfunction theory. Under the indicated condition on the growth of $\alpha_{*}$, it is defined on the space $S_{a}^{1}$, which is nontrivial under this conditionf because it contains the entire function $\prod_{j=1}^{n} e^{-4 \cosh z_{j}}$ majorizable by $e^{-n e^{|x| / n}}$ for $|y|<\pi / 3$. The elements of $S_{a}^{1}$ are analytic in the domains of the form $\{z:|y|<1 / B\}$ and satisfy the bound $|f(z)| \leq\|f\|_{A, B} e^{-\alpha_{*}(|x| / A)}$ in them. Therefore, the formula

$$
(v, f)=\int \mathbf{v}(x+i y) f(x+i y) \mathrm{d} x \quad(y \in V,|y|<1 / B),
$$

where the integral is independent of $y$ by virtue of the Cauchy-Poincaré theorem, defines a continuous linear functional on $S_{a}^{1}$. We let $u$ denote the (inverse) Fourier transform of $v$ and consider its convolution with a test function $g \in S_{1}^{a}$. We have the identity

$$
(u * g)(p)=(u, g(p-\cdot))=\int \mathbf{v}(x+i y) e^{-i p(x+i y)} f(x+i y) \mathrm{d} x
$$

where

$$
f(z)=(2 \pi)^{-n} \int g(-p) e^{-i p z} \mathrm{~d} p .
$$

\footnotetext{
${ }^{4}$ If the function $\alpha_{*}$ increases so fast that for any $N$ there exists an $r_{N}$ such that $\alpha_{*}(r)>e^{N r}$ for $r>r_{N}$, then $S_{a}^{1}$ is trivial. This follows from Theorem 4 in Chap. 2 in [11].
} 
The Fourier transformation establishes a one-to-one and bicontinuous mapping of $S_{1}^{a}$ onto $S_{a}^{1}$, and there exist $A^{\prime}>A$ and $B^{\prime}>B$ such that $\|f\|_{S_{a, A^{\prime}}^{1, B^{\prime}}} \leq C\|g\|_{S_{1, B}^{a, A}}$. Therefore, (23) and (25) imply the inequality

$$
|(u * g)(p)| \leq C_{\epsilon, A}\|g\|_{A, B} \inf _{0<t<1 / B^{\prime}} e^{|p| t-\beta^{*}(t / \epsilon)}
$$

Here the infimum is attained at sufficiently large $s=|p|$ on the interval $(0, \delta)$, where $\delta$ is arbitrarily small. Indeed, suppose the contrary, i.e., that for some $\delta>0$ and for any $N$ there exist $s_{N} \geq N$ and $t_{N} \in\left[\delta, 1 / B^{\prime}\right)$ such that

$$
s_{N} t_{N}-\beta^{*}\left(t_{N}\right)<\inf _{0<t<\delta}\left(s_{N} t-\beta^{*}(t)\right)
$$

The right-hand side does not exceed $s_{N} \delta / 2-\beta^{*}(\delta / 2)$, and consequently we have $N\left(t_{N}-\right.$ $\delta / 2)<\beta^{*}\left(t_{N}\right)-\beta^{*}(\delta / 2)$. In view of the monotonicity of $\beta^{*}$, this implies the absurd inequality $N \delta / 2<\beta^{*}\left(1 / B^{\prime}\right)-\beta^{*}(\delta / 2)$. Therefore, (26) remains valid if the infimum is taken throughout the half-axis and the constant $C_{\epsilon, A}$ is increased if necessary. The equivalence of $\beta$ to a concave function implies its equivalence to $\beta^{* *}$, which can be verified by extending this concave function throughout the axis with the value $-\infty$ for $s<0$ and taking into account that the operation of conjugation is involutory for closed concave functions [10]. Ultimately, we obtain

$$
|(u * g)(p)| \leq C_{\epsilon, A}^{\prime}\|g\|_{A, B} e^{\beta(\epsilon|p|)} .
$$

By Lemma 3 in [1], it follows that the functional $u$ has a unique continuous extension to $\mathcal{E}_{\beta}^{\alpha}$. It remains to show that $V^{*}$ is a carrier cone for this extension. In the simplest case of $V=\mathbb{R}^{n}$ and $V^{*}=\{0\}$, this follows from Lemma 1. Indeed, $\mathbf{v} \in \mathcal{A}_{\alpha_{*}}^{\beta^{*}}\left(\mathbb{R}^{n}\right)$ is an entire function increasing no faster than $C_{\epsilon} \exp \left\{\alpha_{*}(\epsilon|z|)\right.$. Estimating the coefficients $c_{k}$ of the power series that represents this function by means of the Cauchy formula results in $\left|c_{\kappa}\right| \leq C_{\epsilon} \epsilon^{|k|} / a_{|k|}$. The test function $g \in \mathcal{E}_{\beta}^{\alpha}(\{0\})$ is also entire, and it satisfies the inequality

$$
|g(p+i q)| \leq\|g\|_{A} e^{\alpha(2 A|p+i q|)}
$$

whence follows the estimate

$$
\left|\partial^{\kappa} g(0)\right| \leq\|g\|_{A} A^{|| \kappa \mid} \kappa ! \inf _{s>0} s^{-k} e^{\alpha(s)}
$$


In view of the Stirling formula, the relation (12) shows that the functional

$$
u=\sum_{\kappa \in \mathbb{N}^{n}} i^{\kappa} c_{\kappa} \partial^{\kappa} \delta
$$

is defined on $\mathcal{E}_{\beta}^{\alpha}(\{0\})$ and is continuous.

The further argument repeats the proof of Theorem 4 in [9]. Let us consider the case of $V=\mathbb{R}_{+}$and $V^{*}=\overline{\mathbb{R}}_{+}$. We apply Theorem 5 and decompose $u$ into the sum of the functionals $u_{+}$and $u_{-}$carried by the respective half-axes $\overline{\mathbb{R}}_{+}$and $\overline{\mathbb{R}}_{-}$. Performing the Laplace transformation, we obtain $\mathbf{v}(x+i 0)=\mathbf{v}_{+}(x+i 0)+\mathbf{v}_{-}(x-i 0)$, where $\mathbf{v}_{ \pm} \in \mathcal{A}_{\alpha_{*}}^{\beta^{*}}\left(\mathbb{C}_{ \pm}\right)$. By the "edge-of-the-wedge" theorem, there is an entire function serving as an extension for both $\mathbf{v}-\mathbf{v}_{+}$and $\mathbf{v}_{-}$. It increases no faster than $C_{\epsilon} \exp \left\{\alpha_{*}(\epsilon|z|)\right.$, and the origin is therefore a carrier cone of the functional $u-u_{+}$, which completes the proof for $n=1$. In the general case, it can be assumed that the first coordinate unit vector lies in the cone $V$. We take $g \in \mathcal{E}_{\beta}^{\alpha}\left(\mathbb{R}^{n-1}\right)$, write $x^{\prime}=\left(x_{2}, \ldots, x_{n}\right)$, and consider the mapping

$$
g \rightarrow \mathbf{v}_{1}\left(z_{1}\right)=\int \mathbf{v}\left(z_{1}, x^{\prime}\right) \check{g}\left(x^{\prime}\right) \mathrm{d} x^{\prime}
$$

of $\mathcal{E}_{\beta}^{\alpha}\left(\mathbb{R}^{n-1}\right)$ into $\mathcal{A}_{\alpha_{*}}^{\beta^{*}}\left(\mathbb{C}_{+}\right)$. It is easy to see that it is continuous. Let $u_{1} \in \mathcal{E}_{\beta}^{\prime \alpha}\left(\overline{\mathbb{R}}_{+}\right)=$ $\mathcal{E}_{\beta}^{\prime \alpha}\left(\mathbb{R}_{+}\right)$be the functional that is taken to $\mathbf{v}_{1}$ by the Laplace transformation. The correspondence $\mathbf{v}_{1} \rightarrow u_{1}$ is also continuous by the open mapping theorem (see Sec. 5.6 in [7]). We thus obtain a bilinear separately continuous functional on $\mathcal{E}_{\beta}^{\alpha}\left(\mathbb{R}_{+}\right) \times \mathcal{E}_{\beta}^{\alpha}\left(\mathbb{R}^{n-1}\right)$, which determines by Theorem 3 a continuous linear functional on $\mathcal{E}_{\beta}^{\alpha}\left(H_{1}\right)$, where $H_{1}$ is the half-space $x_{1}>0$. The restriction of this functional to $\mathcal{E}_{\beta}^{\alpha}(\mathbb{R}) \otimes \mathcal{E}_{\beta}^{\alpha}\left(\mathbb{R}^{n-1}\right)$ coincides with that of the functional $u$, and we conclude that $\bar{H}_{1}$ is a carrier cone for $u$. The same is true for each of the half-spaces $\{p: p y \geq 0\} \quad y \in V$ with $y \in V$, whose intersection is the cone $V^{*}$, which consequently is also a carrier cone of the functional $u$ by Theorem 6 . This completes the proof.

It is also useful to have a theorem characterizing spectral functions in the case of preliminarily unknown regularity properties for the growth of holomorphic functions near the real boundary of the analyticity domain. The statement below is suitable for our aims.

Theorem 9. Suppose $\mathbf{v}$ is a holomorphic function in $T^{V}$, and for any compact subcone $V^{\prime}$ of $V$ and for an arbitrary $\epsilon>0$ the inequality

$$
|\mathbf{v}(z)| \leq C_{\epsilon}\left(V^{\prime}\right) \exp \left\{\alpha_{*}(\epsilon|z|)+\gamma_{V^{\prime}}(|y|)\right\} \quad\left(y \in V^{\prime}\right)
$$


holds, where $\gamma_{V^{\prime}}$ is a nonnegative function indefinitely increasing with decreasing argument and $\alpha_{*}$ is conjugate to a function $\alpha$ possessing the properties specified in Definition 1 and increases no faster than a linear exponential function. Then $\mathbf{v}$ is the Laplace transform of an analytic functional belonging to any space $\mathcal{E}_{\beta}^{\prime \alpha}\left(V^{*}\right)$, where $\beta$ satisfies (20) and the inequality

$$
\left(-\gamma_{V^{\prime}}\right)^{*}(s) \leq C_{\epsilon}^{\prime}\left(V^{\prime}\right)+\beta(\epsilon s)
$$

Indeed, substituting (28) in (25), we obtain an estimate of the type (26) with $\gamma_{V^{\prime}}(t)$ instead of $-\beta^{*}(t / \epsilon)$. As before, the monotonicity of $\gamma_{V^{\prime}}$ permits passing to the infimum throughout the half-axis in this estimate, which yields the inequality (27) by virtue of (29). The remaining part of the proof is the same as in Theorem 8 in view of the inequality $\gamma_{V^{\prime}}(t) \leq C_{\epsilon}^{\prime}\left(V^{\prime}\right)-\beta^{*}(t / \epsilon)$ following from $(29)$.

\section{Generalized spectral condition}

The functional domain of definition of the Wick series $\sum d_{k}: \phi^{k}:(x)$ was found in [1] proceeding from the infrared and ultraviolet behavior of the Hilbert majorant of the vacuum expectation value $w\left(x-x^{\prime}\right)=\left\langle\Psi_{0}, \phi(x) \phi\left(x^{\prime}\right) \Psi_{0}\right\rangle$. The majorant is the boundary value of a function $\mathbf{w}_{\text {maj }}\left(z, z^{\prime}\right)$ holomorphic in the tubular domain $\left\{\left(z, z^{\prime}\right) \in \mathbb{C}^{2 \mathrm{~d}}: y=\right.$ $\left.\operatorname{Im} z \in \mathbb{V}_{-}, y^{\prime}=\operatorname{Im} z^{\prime} \in \mathbb{V}_{+}\right\}$, and its behavior can be characterized by the inequality

$$
\left|\mathbf{w}_{\text {maj }}\left(z, z^{\prime}\right)\right| \leq C_{0}+C_{1} w_{I R}\left(|z|+\left|z^{\prime}\right|\right)+C_{2} w_{U V}\left(|y|+\left|y^{\prime}\right|\right)
$$

where the pair $\left(y, y^{\prime}\right)$ runs through a compact subcone of $\mathbb{V}_{-} \times \mathbb{V}_{+}$, the constant $C_{2}$ depends on this subcone, and $w_{I R}$ and $w_{U V}$ are nonnegative monotonic functions, the first of them increasing, and the other decreasing. The inequality (30) was used in [1] only for $y$ and $y^{\prime}$ belonging to the $y_{0}$-axis, whereas here we need its satisfaction to the full extent.

Let the series $\sum d_{k}: \phi^{k}:(x)$ be convergent under averaging with test functions belonging to $S_{a}^{b}=\mathcal{F}\left(\mathcal{E}_{\beta}^{\alpha}\right)$. By the generalized spectral condition, we mean the requirement that the closed cone (5) be a carrier cone of the vector-valued analytic functional (2) in the momentum representation. Let $\check{f} \in \mathcal{E}_{\beta}^{\alpha}\left(K_{n-}\right)$, let $\check{f}_{\nu} \in \mathcal{E}_{\beta}^{\alpha}$, and let $\check{f}_{\nu} \rightarrow \check{f}$. To

prove the validity of the stated condition, it suffices to show that $\Psi\left(f_{\nu}\right)=\hat{\Psi}\left(\check{f}_{\nu}\right)$ is a Cauchy sequence. This is true if the acute cone $\left(-K_{n-}\right) \times K_{n-}$ is a carrier cone of the 
Fourier transform of the generalized function $T_{n}$ in the right-hand side of (11). Theorem 9 reduces deriving this property to estimating the behavior of the corresponding analytic function $\mathbf{T}_{n}(z)$. It should be taken into consideration here that the transition from the momentum representation to the coordinate representation in quantum field theory is usually realized with the opposite sign in the Fourier-Laplace transformation as compared with the one used in the foregoing section. Explicitly, we have $\mathbf{T}_{n}(z)=\sum_{K} D_{K} \mathbf{W}^{K}(z)$, where $z \in \mathbb{C}^{2 n d}, K$ is a multi-index with the components $k_{j m}$, the coefficients $D_{K}$ can be expressed via $d_{k}$ in a known way, and

$$
\mathbf{W}^{K}(z)=\prod_{1 \leq j<m \leq n} \mathbf{w}\left(z_{m}-z_{j}\right)^{k_{j m}} \prod_{n+1 \leq j<m \leq 2 n} \mathbf{w}\left(z_{j}-z_{m}\right)^{k_{j m}} \prod_{\substack{1 \leq j \leq n \\ n+1 \leq m \leq 2 n}} \mathbf{w}_{\mathrm{maj}}\left(z_{j}, z_{m}\right)^{k_{j m}} .
$$

We write $V_{n-}=\operatorname{int} K_{n-}^{*}$ and $V=V_{n-} \times\left(-V_{n-}\right)$. Then

$$
\begin{aligned}
V=\left\{y \in \mathbb{R}^{2 n \mathrm{~d}}: y_{1} \in \mathbb{V}_{-}, \quad y_{j}-y_{j-1} \in \mathbb{V}_{-}(2 \leq j \leq n),\right. \\
\left.y_{n+1} \in \mathbb{V}_{+}, \quad y_{j}-y_{j-1} \in \mathbb{V}_{+}(n+2 \leq j \leq 2 n)\right\} .
\end{aligned}
$$

Let a cone $V^{\prime}$ be compact in $V$, let $1 \leq j \leq n$, and let $n+1 \leq m \leq 2 n$. If $y$ ranges $V^{\prime}$, then the pair $\left(y_{j}, y_{m}\right)$ ranges a compact subcone of $\mathbb{V}_{-} \times \mathbb{V}_{+}$, and there is a $\delta>0$ such that $\left|y_{j}\right|+\left|y_{m}\right| \geq \delta|y|$. Indeed, let pr $V^{\prime}$ denote the projection of the cone $V^{\prime}$, i.e., its intersection with the unit sphere in $\mathbb{R}^{2 n d}$, and let the expression $\left|y_{j}\right|+\left|y_{m}\right|$ be regarded as a function of $y$. This function is continuous, maps the compact set pr $V^{\prime}$ into a compact subset in $\mathbb{V}_{-} \times \mathbb{V}_{+}$, and attains its infimum on pr $V^{\prime}$, which is nonzero because points with zero components do not belong to $V$. This precisely implies the desired assertion. A similar argument shows that for the indices $1 \leq j<m \leq n$ and $n+1 \leq j<m \leq 2 n$, the difference $y_{j}-y_{m}$ between the components of the vector $y \in V^{\prime}$ ranges compact subcones of $\mathbb{V}_{+}$and $\mathbb{V}_{-}$respectively, and we have $\left|y_{j}-y_{m}\right| \geq \delta|y|$, possibly with a different, but nonzero, constant $\delta$. Applying the inequality $\left|\mathbf{w}\left(x-x^{\prime}-2 i \eta\right)\right|^{2} \leq\left|\mathbf{w}_{\text {maj }}(x-i \eta, x+i \eta)\right|\left|\mathbf{w}_{\text {maj }}\left(x^{\prime}-i \eta, x^{\prime}+i \eta\right)\right|$ proved in [1], which holds for all $\eta \in \mathbb{V}_{+}$, using (30), and taking into account the monotonicity of $w_{I R}$ and $w_{U V}$, we obtain

$$
\left|\mathbf{W}^{K}(z)\right| \leq 3^{|K|}\left(C_{0}^{|K|}+C_{1}^{|K|} w_{I R}(2|z|)^{|K|}+C_{2}\left(V^{\prime}\right)^{|K|} w_{U V}\left(\delta_{V^{\prime}}|y|\right)^{|K|}\right) \quad y \in V^{\prime} .
$$

We subject the coefficients of the Wick series in question to the conditions (whose meaning was elucidated in [1])

$$
d_{k} \geq 0, \quad d_{0}=1, \quad \lim _{k \rightarrow \infty}\left(k ! d_{k}^{2}\right)^{1 / k}=0, \quad d_{k} d_{l} \leq C H^{k+l} d_{k+l}
$$


where $C$ and $H$ are constants. The third condition in (32) ensures the absolute and uniform convergence of the series representing the function $\mathbf{T}_{n}(z)$ on compact sets in $T^{V}$ and, as a consequence, the holomorphy of this function in $T^{V}$, whereas the fourth condition permits deriving from (31) the inequality

$$
\left|\mathbf{T}_{n}(z)\right| \leq C\left(\sum_{k=0}^{\infty} L^{k} k ! d_{2 k} w_{I R}(2|z|)^{k}\right)\left(\sum_{k=0}^{\infty} L^{k} k ! d_{2 k} w_{U V}(\delta|y|)^{k}\right), \quad y \in V^{\prime}
$$

where $L$ and $\delta$ depend $V^{\prime}$.

We can now summarize our results.

Theorem 10. Let $\phi$ be a free field acting in a pseudo-Hilbert space $\mathcal{H}$. Under the conditions (32) on the coefficients, the field $\varphi(x)=\sum d_{k}: \phi^{k}:(x)$ is well defined as an operator-valued distribution on every space $S_{a}^{b}$ whose indicator functions satisfy the inequalities

$$
\sum_{k} L^{k} k ! d_{2 k} w_{I R}(r)^{k} \leq C_{L, \epsilon} a(\epsilon r), \quad \inf _{t>0} e^{s t} \sum_{k} L^{k} k ! d_{2 k} w_{U V}(t)^{k} \leq C_{L, \epsilon} b(\epsilon s)
$$

for an arbitrarily large $L>0$ and an arbitrarily small $\epsilon>0$, and, in addition, $\log a(r)$ grows no faster than a linear exponential function. If $S_{b}^{a}$ is a Gelfand-Shilov-Gurevich space and $\beta(s)=\log b(s)$ satisfies (20), then all conditions of the pseudo-Wightman formalism hold for $\varphi(x)$ including the generalized spectral condition.

The first of these assertions was proved in [1], and the other can be derived from (33) using Theorem 9 if $\gamma_{V^{\prime}}(t)=\ln \sum_{k} L_{V^{\prime}}^{k} k ! d_{2 k} w_{U V}\left(\delta_{V^{\prime}} t\right)^{k}$ is taken.

\section{Conclusion}

The functional domain of definition of the Wick series of a free field with an indefinite metric was found comparatively simply in [1]. In contrast, proving that the basic conditions of general quantum field theory are satisfied for sums of such series requires studying the properties of analytic functionals rather thoroughly. We believe that these efforts are justified because they not only permit properly refining the pseudo-Wightman formalism but also, in our opinion, provide a mathematical basis for a consistent Euclidean formulation of quantum field models with singular infrared behavior violating the positivity condition. 
The above condition on the growth of the indicator function $a(r)$ is not essential and is motivated only by the desire to give clearer, more concise proofs. We note that the Wick series in question can always be realized on the spaces $S_{0}^{b}$, which correspond to the spaces $\mathcal{E}_{\beta}^{\alpha}$ with $\alpha(s)=s$. These spaces allow arbitrary infrared behavior because they consist of functions of compact support in the coordinate representation, and the corresponding formulation of the spectral condition is the weakest (i.e., the most general). However, the objective of the present paper is to accurately describe both the functional domain of definition of Wick series and their spectral properties. The developed technique permits solving another interesting problem completely, namely, investigating the nonlocal extension of the Borchers equivalence classes, in which the major difficulty is related to correctly generalizing the microcausality condition and proving that it is satisfied for nonlocal Wick series. These results will be presented in a separate publication.

Acknowledgments. This work was supported by the Russian Foundation for Basic Research (Grant No. 99-02-17916) and INTAS (Grant No. 99-1-590).

\section{References}

[1] A. G. Smirnov and M. A. Soloviev, Theor. Math. Phys., 123, 709 (2000), math$\mathrm{ph} / 0010001$.

[2] N. N. Bogoliubov, A. A. Logunov, A. I. Oksak, and I. T. Todorov, General Principles of Quantum Field Theory [in Russian], Nauka, Moscow (1987); English transl., Kluwer, Dordrecht (1990).

[3] U. Moschella and F. Strocchi, Lett. Math. Phys., 24, 103 (1992).

[4] U. Moschella, J. Math. Phys., 34, 535 (1993).

[5] M. A. Soloviev, Theor. Math. Phys., 105, 1520 (1995).

[6] I. M. Gelfand and G. E. Shilov, Generalized Functions [in Russian], Vol. 2, Function and Generalized Function Spaces, Gos. Izd. Fiz.-Mat. Lit., Moscow (1958); English transl., Acad. Press, New York (1968). 
[7] M. Reed and B. Simon, Methods of Modern Mathematical Physics, Vol. 1, Functional Analysis, Acad. Press, New York (1972).

[8] M. A. Soloviev, Lett. Math. Phys., 33, 49, (1995), hep-th/9403083.

[9] M. A. Soloviev, Commun. Math. Phys., 184, 579 (1997), hep-th/9601005.

[10] R. T. Rockafellar, Convex Analysis, Princeton Univ. Press, Princeton, NJ (1970).

[11] S. Mandelbrojt, Closure Theorems and Composition Theorems [in Russian] (Lectures at Moscow State University transcribed and translated by E. A. Gorin, A. S. Dynin, and B. S. Mityagin and edited using the monographs by S. Mandelbrojt, General Theorems of Closure (Rice Inst. Pamphlet, Spec. issue, Nov. 1951) and Composition Theorems (Rice Inst. Pamphlet, 45, 3, Oct. 1958)), Izd. Inostr. Lit., Moscow (1962). 